\title{
Tidally Induced Cross-Frontal Mean Circulation: Analytical Study*
}

\author{
Changming Dong, Hsien-Wang Ou, Dake Chen, and Martin Visbeck \\ Lamont-Doherty Earth Observatory, Columbia University, Palisades, New York
}

(Manuscript received 5 December 2002, in final form 19 June 2003)

\begin{abstract}
An analytical model is developed to study the tidally induced mean circulation in the frontal zone. Four distinct forcing mechanisms are identified, which result in the generation of the counterclockwise Bernoulli cell, the clockwise Ekman cell, the clockwise frontal cell, and the Stokes drift (facing in the direction with the shallow water to the left). The decomposition of the cross-frontal circulation provides a dynamical framework for interpreting and understanding its complex structure. To illustrate the underlying physics, three model configurations are considered pertaining to a homogenous ocean and winter and summer fronts. For a homogeneous ocean, the circulation is dominated by three cells; for the winter front, the offshore Bernoulli cell is strengthened; and for the summer front, two counterrotating cells are found in the vertical direction, associated with the two branches of the front. The dependence of the cell structure on the Ekman, Burger, and other dimensionless numbers is examined.
\end{abstract}

\section{Introduction}

There are two components of the flow in a tidal frontal zone: alongfrontal and cross-frontal ones. The alongfrontal component, being dominated by the thermalwind shear, is better understood and may account for the summer intensification of the clockwise gyre over the Georges Bank (Loder and Wright 1985; Ou 1999, 2000). The cross-frontal component, being much weaker, is less well understood. The progress is further hampered by the difficulty in discerning the flow in the noisy environment where the front itself undergoes a large excursion. Despite the relative weakness generally associated with the cross-frontal circulation, the importance of these flows in the practical application cannot be ignored. In fact, the tidal-induced upwelling and downwelling, which produce vertical transport of nutrient and other materials important for biological productivity and sediment transport, are caused by these flows (Tee 1985).

In general terms, the tidal-induced cross-frontal circulation can be divided into two components: the one driven by the tidal rectification and the other by the buoyancy force associated with density variation. The two are referred to here as the nonfrontal and the frontal components, respectively.

* Lamont-Doherty Earth Observatory Contribution Number 6530.

Corresponding author address: Changming Dong, Program in Atmospheric and Oceanic Sciences, Princeton University, Sayre Hall, Princeton, NJ 08544.

E-mail: cdong@splash.princeton.edu
Previous studies show that multiple cells are the basic structure of such circulation (Loder 1980; Tee 1985; Loder and Wright 1985; Chen and Beardlsey 1995a,b, 1998). With a depth-averaged tidal model, Loder (1980) suggested that the cross-shore circulation consist of multiple cells in response to the mean surface elevation. Greenberg (1983) reached a similar conclusion using a two-dimensional depth-averaged numerical tidal model.

Using a three-dimensional circulation model, Tee (1985) calculated the mean circulation over the Georges Bank. Over its northern flank, the cross-shore circulation is composed of three parts (viewed with the deep water to the left): a counterclockwise cell in the deep water, a clockwise cell in the middle shelf, and the on-bank flow at all depths on the shallow side of the front. A similar structure is found over the southern flank, except that the middle clockwise cell is weaker and the flow is off-bank in the shallow water. The buoyancy field is used in determining the vertical viscosity through a Richardson number.

Using an analytical tidal rectification model with a rigid lid and a depth-independent eddy viscosity, Loder and Wright (1985) computed the cross-frontal circulation over the Georges Bank. The model predicted that the nonfrontal component associated with the tidal rectification on both sides of the Georges Bank generally consists of two opposite-directed cells, with the bottom current directed off-bank in the shallow-water cell and on-bank in the other. The model also predicted that the frontal component associated with the density fields is dominated by a cell with on-bank bottom flow.

Chen and Beardsley (1995a,b, 1998) applied the 
Princeton Ocean Model to study the cross-frontal circulation, which also has a multiple-cell structure. Their analysis of momentum balance for the mean motions suggested that it is driven by the tidal rectification, as modified by the vertical diffusion of the alongfrontal flow (in the alongshore direction, the Coriolis force, which represents the cross-shore current, is balanced by the tidal rectification and vertical diffusion of the alongfrontal current).

The multiple-cell structure in the cross-frontal circulation is also suggested in the field data. A suite of ADCP observations for 2-3 July 1988 on the northern flank of Georges Bank provided a cross-frontal mean circulation (Loder and Brickman 1992; Loder et al. 1993). It shows that in shallow water the cross-shore flow is on-bank and in deep water the flow is on-bank near the top and the bottom and off-bank in the interior, suggesting a multiple-cell structure. Passive-tracer experiments by Houghton and Ho (2001) show that the dye patch moves onshore near the bottom and offshore at middepths, which indicates multiple-cell structure in the Lagrangian view.

Although the aforementioned modeling studies have made substantial progress toward interpreting the crossfrontal circulation, the multiple cells have not been explained within a dynamical framework. Such an explanation constitutes the main contribution of the present work.

To elucidate the basic physics, we consider oceans that are homogenous and contain a front, respectively. The latter case is further divided into winter and summer fronts. Because of the different origins of the two types of fronts, their basic structures are different. A winter front marks the boundary between the fresher near-shore water and the saltier offshore water, which extends from the bottom to the surface. In the summertime, the seasonal thermocline is formed because of surface heat. In the shallow water, the stratification is destroyed by strong mixing, and then a summer front is formed with two branches, which intersect both top and bottom surfaces, respectively (Hill et al. 1993). In this study, the basic structures of winter and summer fronts are taken from Ou et al. (2003) and Simpson and Hunter (1974), respectively.

This paper is organized as follows: In section 2, the analytical model for the cross-frontal mean circulation is deduced. In section 3, the forcing mechanisms that drive the circulation are discussed. In section 4 , the analytical solution is presented and three basic cases of homogenous ocean and winter and summer fronts are discussed. In section 5 , the sensitivity of the analytical solution to model parameters is examined. Sections 6 and 7 are the discussion and conclusions, respectively.

\section{Model description}

The model contains assumptions similar to those of Wright and Loder (1985), which include the following:
1) the current is dominated by a single tidal constituent (at frequency $\sigma$ ), 2) the mean flow is sufficiently weak that its influence on the tidal flow is negligible (weak nonlinearity), 3) the vertical force balance is hydrostatic, 4) no baroclinic pressure gradients are at the tidal frequency, 5) the bottom topography and all variables are uniform along the shore, 6) vertical viscosity is constant, and 7) as a diagnostic model, the density field is prescribed and its development is not considered. Note that assumption 4 allows the tidal currents to be computed as if the ocean were homogenous.

Based on the above assumptions, the equations governing the mean flows are

$$
\begin{aligned}
\mathrm{TSU}^{*}-f \bar{v}^{*} & =-g \frac{\partial \bar{\eta}}{\partial x}-\frac{1}{\rho_{0}} \frac{\partial \bar{p}_{\rho}^{*}}{\partial x^{*}}+\nu \frac{\partial^{2} \bar{u}^{*}}{\partial z^{* 2}}, \\
\mathrm{TSV}^{*}+f \bar{u}^{*} & =\nu \frac{\partial^{2} \bar{v}^{*}}{\partial z^{* 2}} \\
\frac{\partial \bar{u}^{*}}{\partial x^{*}}+\frac{\partial \bar{w}^{*}}{\partial z^{*}} & =0, \text { and } \\
\frac{\partial \bar{p}^{*}}{\partial z^{*}} & =-\bar{\rho}^{*} g
\end{aligned}
$$

where

$$
\begin{aligned}
\mathrm{TSU}^{*} & =\overline{u^{\prime *} \frac{\partial u^{\prime *}}{\partial x^{*}}}+\overline{w^{\prime * \frac{\partial u^{\prime *}}{\partial z^{*}}}} \text { and } \\
\mathrm{TSV}^{*} & =\overline{u^{\prime *} \frac{\partial v^{\prime *}}{\partial x^{*}}}+\overline{w^{\prime * \frac{\partial v^{\prime *}}{\partial z^{*}}}}
\end{aligned}
$$

are so-called tidal stresses and a right-handed Cartesian coordinate system $(x, y, z)$ is used, with the $x$ axis pointing offshore, the $y$ axis pointing alongshore with the shallow area to its left, and the $z$ axis increasing upward from the sea surface. The lowercase $(u, v, w)$ denote the cross-front, alongfront, and vertical current, respectively; $g$ is the gravitational acceleration, $f$ is the Coriolis coefficient, $\nu$ is the vertical eddy diffusivity coefficient, $\eta$ is the surface elevation, $p_{\rho}$ is the pressure associated with the density variation, and $\rho_{0}$ is the static density. An asterisk represents a dimensional parameter; a variable with no asterisk is dimensionless except for these parameters: $f, g$, and $\nu$. The prime denotes the tidal component. An overbar denotes time averaging over a tidal period. The tidal components can be solved analytically (see appendix A), which solution can be substituted into the above equation.

The boundary conditions at the surface $z^{*}=0$, where no wind stress is applied, are

$$
\frac{\partial\left(\bar{u}^{*}, \bar{v}^{*}\right)}{\partial z^{*}}=0,
$$

and at the bottom $z^{*}=-h^{*}$, a linear stress condition is applied, 

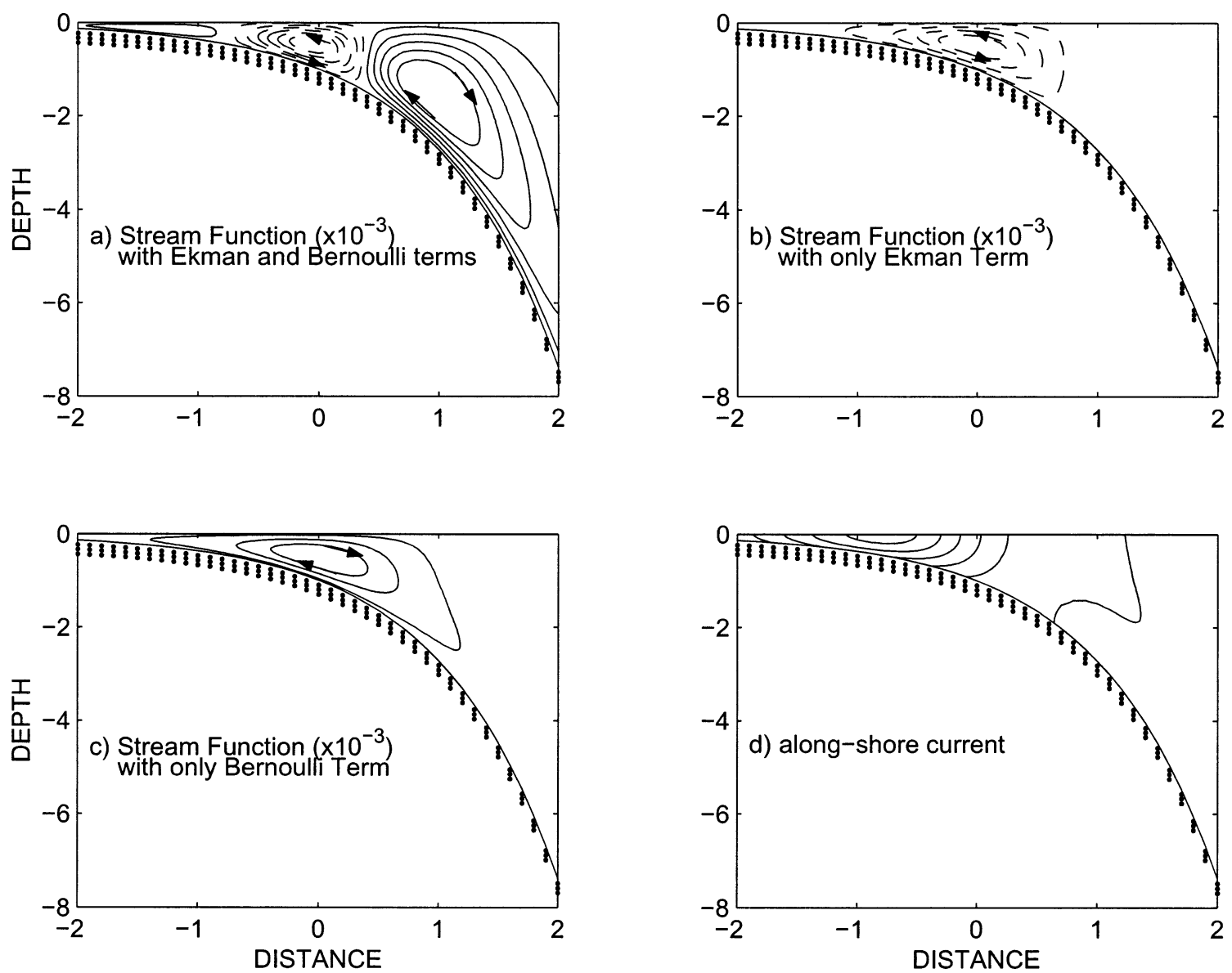

FIG. 1. The nondimensional streamfunction for a homogenous ocean. (a) The cross-shore streamfunction with Ekman and Bernoulli terms. Contour interval is $1.1 \times 10^{-3}$, and dotted lines are for values less than zero. (b) Cross-shore streamfunction with only Ekman term. Contour interval is $7.0 \times 10^{-3}$. (c) Streamfunction with only Bernoulli term. Contour interval is $7.0 \times 10^{-3}$. (d) Alongshore current with Ekman and Bernoulli term. Contour interval is 0.1 , and negative value represents the flow pointing out of the page.

$$
v \frac{\partial\left(\bar{u}^{*}, \bar{v}^{*}\right)}{\partial z^{*}}=k\left(\bar{u}^{*}, \bar{v}^{*}\right)
$$

The above equations can be nondimensionalized, with the scaling denoted by the square bracket:

$$
\begin{aligned}
{\left[\bar{u}^{*}\right],\left[\bar{v}^{*}\right] } & =R_{O} E_{V} U_{0}, \\
{\left[\bar{w}^{*}\right] } & =\left[\bar{u}^{*}\right] \frac{H_{0}}{L}, \\
{\left[\Delta \bar{\eta}^{*}\right] } & =\frac{\rho_{0} U_{0}^{2}}{g}, \text { and } \\
{\left[\Delta \bar{p}_{\rho}^{*}\right] } & =B_{U} \rho_{0} U_{0}^{2},
\end{aligned}
$$

where

$$
\begin{array}{ll}
E_{V}=\frac{\nu}{f H_{0}^{2}} & \text { (Ekman number), } \\
R_{O}=\frac{U_{0}}{f L} & \text { (Rossby number), }
\end{array}
$$

$$
B_{U}=\frac{\Delta \rho g H_{0}}{\rho_{0} U_{0}^{2}} \quad \text { (Burger number), }
$$

and $U_{0}, H_{0}$, and $L$ represent the scales of the amplitude of tidal current, the water depth, and the cross-shore scale, respectively. Note that the mean circulation is displayed in the same scale as the tidal current for convenient comparison with tide currents. With the nondimensionalization, one obtains

$$
\begin{aligned}
\mathrm{TSU}-E_{V} \bar{v} & =-B_{U} \frac{\partial \bar{p}_{\rho}}{\partial x}-\frac{\partial \bar{\eta}}{\partial x}+E_{V}^{2} \frac{\partial^{2} \bar{u}}{\partial z^{2}}, \\
\mathrm{TSV}+E_{V} \bar{u} & =E_{V}^{2} \frac{\partial^{2} \bar{v}}{\partial z^{2}}, \quad \text { and } \\
\frac{\partial \bar{u}}{\partial x}+\frac{\partial \bar{w}}{\partial z} & =0 .
\end{aligned}
$$

The corresponding boundary conditions for the nondimensional equations become 

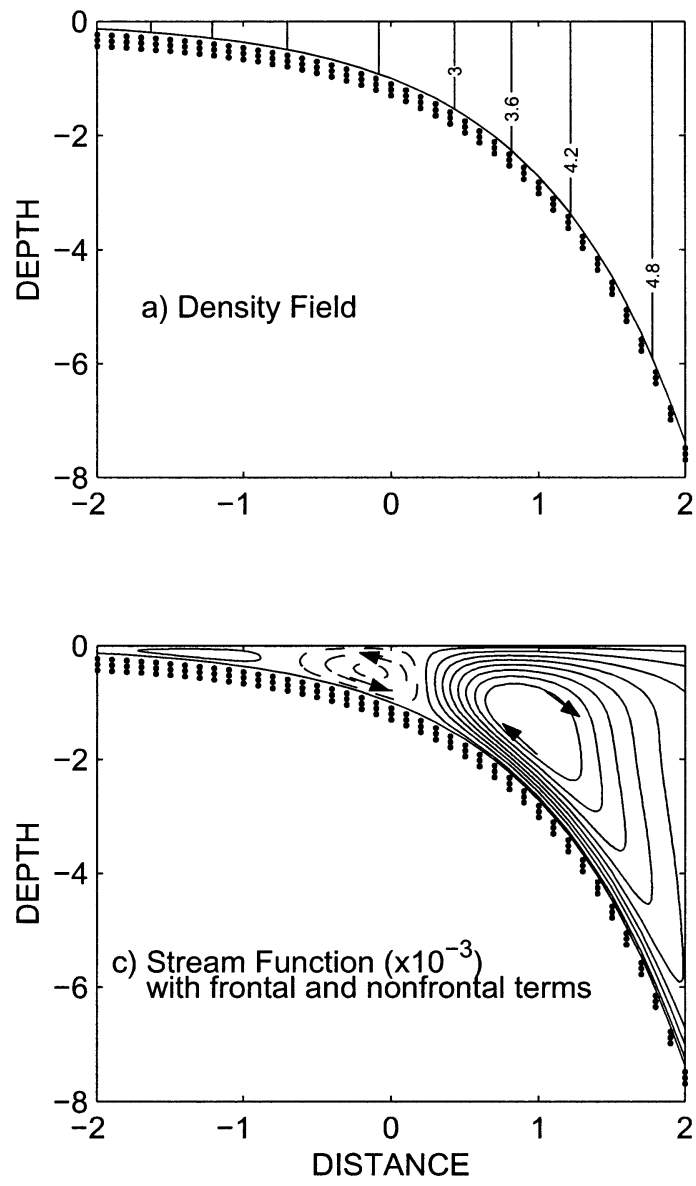

$$
\begin{array}{ll}
\frac{\partial(\bar{u}, \bar{v})}{\partial z}=0 & \text { at } z=0 \quad \text { and } \\
\frac{\partial(\bar{u}, \bar{v})}{\partial z}=\frac{F}{E_{V}}(\bar{u}, \bar{v}), & \text { at } z=-h,
\end{array}
$$

where

$$
F=\frac{k}{f H_{0}} .
$$

In Wright and Loder (1985), the above equations are solved directly. In the present model, with the aim of illustrating the driving mechanisms for the cross-frontal circulation, we introduce the streamfunction $\psi$ from the continuity equation (2.18):

$$
\begin{aligned}
& \frac{\partial \psi}{\partial z}=-\bar{u} \quad \text { and } \\
& \frac{\partial \psi}{\partial x}=\bar{w},
\end{aligned}
$$

and, integrating (2.22) from the surface to the level $z$, one has

$$
\psi(z)=\psi(0)-\int_{0}^{z} \bar{u} d z
$$

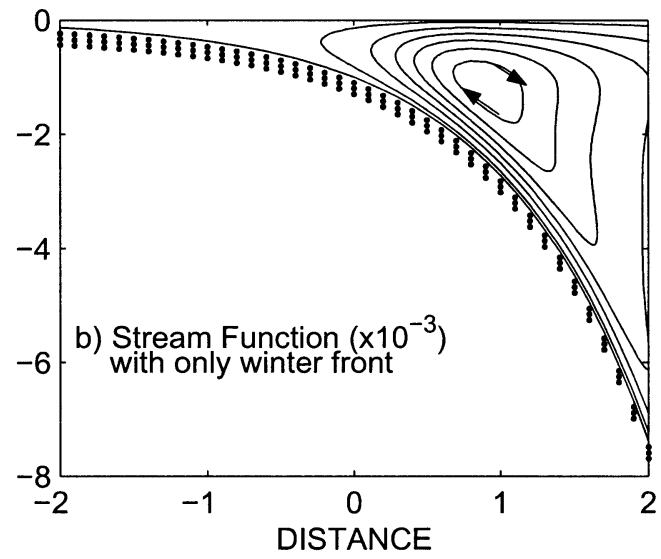

FIG. 2. (a) The density structure of winter front, and (b) crossfrontal streamfunction with the winter front only. (c) Cross-frontal streamfunction with all of frontal, Ekman, and Bernoulli terms. Contour interval is $2.0 \times 10^{-3}$, and dotted lines are for values less than zero

Integrating (2.17) from the surface to level $z$, substituting $\bar{v}$ from (2.16), and using (2.24) and the boundary conditions (2.19), one obtains

$$
\begin{aligned}
\psi(z)+E_{V}^{2} \frac{\partial^{4} \psi}{\partial z^{4}}= & B_{U} \frac{\partial \bar{\rho}}{\partial x}-\frac{\partial(\mathrm{TSU})}{\partial z} \\
& +\frac{1}{E_{V}} \int_{0}^{z}(\mathrm{TSV}) d z+\psi(0) .
\end{aligned}
$$

The magnitude of $\psi(0)$ can be linked to the Stokes drift through the following consideration. In (2.24), at $z=-h$, if the streamfunction along the bottom is set to be zero, one has

$$
\psi(0)=-\int_{-h}^{0} \bar{u} d z .
$$

To the first approximation (Longuet-Higgins 1969), the Lagrangian velocity (subscripted " $L$ ") is the sum of the Eulerian velocity and the Stokes drift (subscripted " $S$ '):

$$
\bar{u}_{L}=\bar{u}+u_{S} \text {. }
$$

If one integrates the expression vertically and notes that the Lagrangian transport is zero, which implies no mass accumulation, one has 

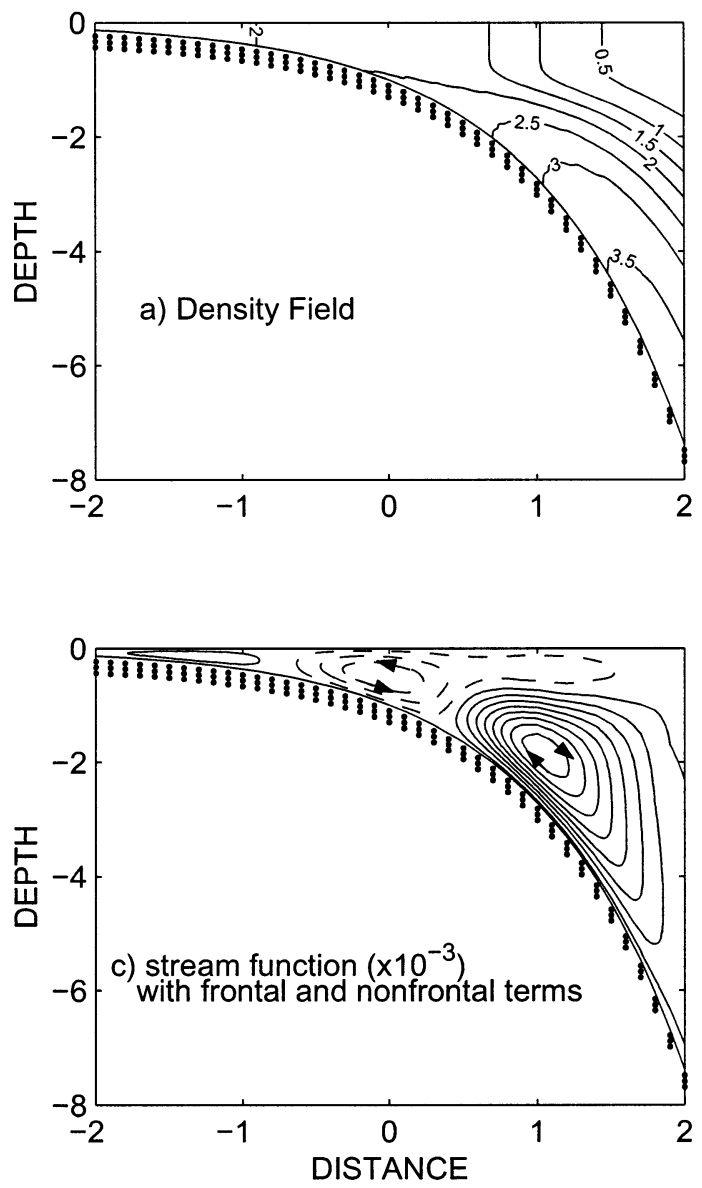

$$
\int_{-h}^{0} \bar{u} d z=-\int_{-h}^{0} u_{S} d z
$$

According to Longuet-Higgins (1969),

$$
\int_{-h}^{0} u_{S} d z=\frac{M}{r E_{V}} \overline{\eta^{\prime}(x) u^{\prime}(x, 0)} \equiv Q_{S},
$$

where $M$ is a dimensionless parameter, the square of the ratio of the topography horizontal scale to the tidal wavelength, and $r$ is the ratio of the Coriolis parameter to the tidal frequency. Both definitions can be found in appendix A.

Combining (2.26), (2.28), and (2.29), one has

$$
\psi(0)=Q_{S} .
$$

Expressed in terms of the streamfunction, the boundary conditions become

$$
\begin{array}{lll}
\psi=Q_{S} \quad \text { and } \quad \frac{\partial^{2} \psi}{\partial z^{2}}=0 & \text { at } z=0 \text { and } \\
\psi=0 & \text { and } \quad \frac{\partial^{2} \psi}{\partial z^{2}}=\frac{F}{E_{V}} \frac{\partial \psi}{\partial z} & \text { at } z=-h .
\end{array}
$$

Solving (2.25) with boundary conditions (2.31) and

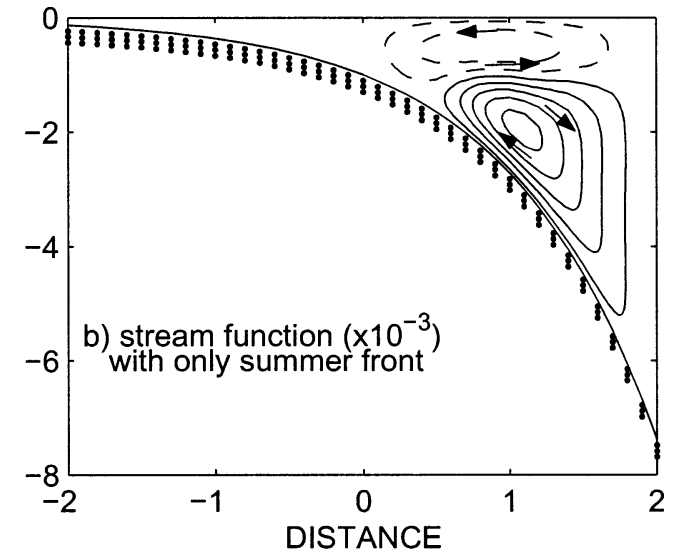

FIG. 3. Same as in Fig. 2, but for summer front.

(2.32), one can obtain the cross-frontal mean streamfunction.

\section{Forcing mechanisms}

Before solving (2.25), we first examine its physical contents. Four terms on the right side of (2.25) are identified as forcing terms and are discussed in the following. The first term represents the buoyancy force associated with the density field. Given a vertical density front, it acts to generate a cell, referred as the "frontal cell." It is in the same sense as the gravitational tendency, except it is affected through thermal wind because of the earth's rotation.

The second term stems from spatial variation of the tidal kinetic energy, whose sign is more varied. Because the tidal amplitude generally increases upward and shoreward, this term is positive over the bulk of the water column; close to the bottom, however, it is negative because of the bottom friction. The term is related to the spatial variation of the kinetic energy, whose physical meaning could be understood with the Bernoulli law: that is, horizontal variation of the kinetic energy gives rise to pressure gradient, whose vertical variation then generates the vertical shear. The impor- 

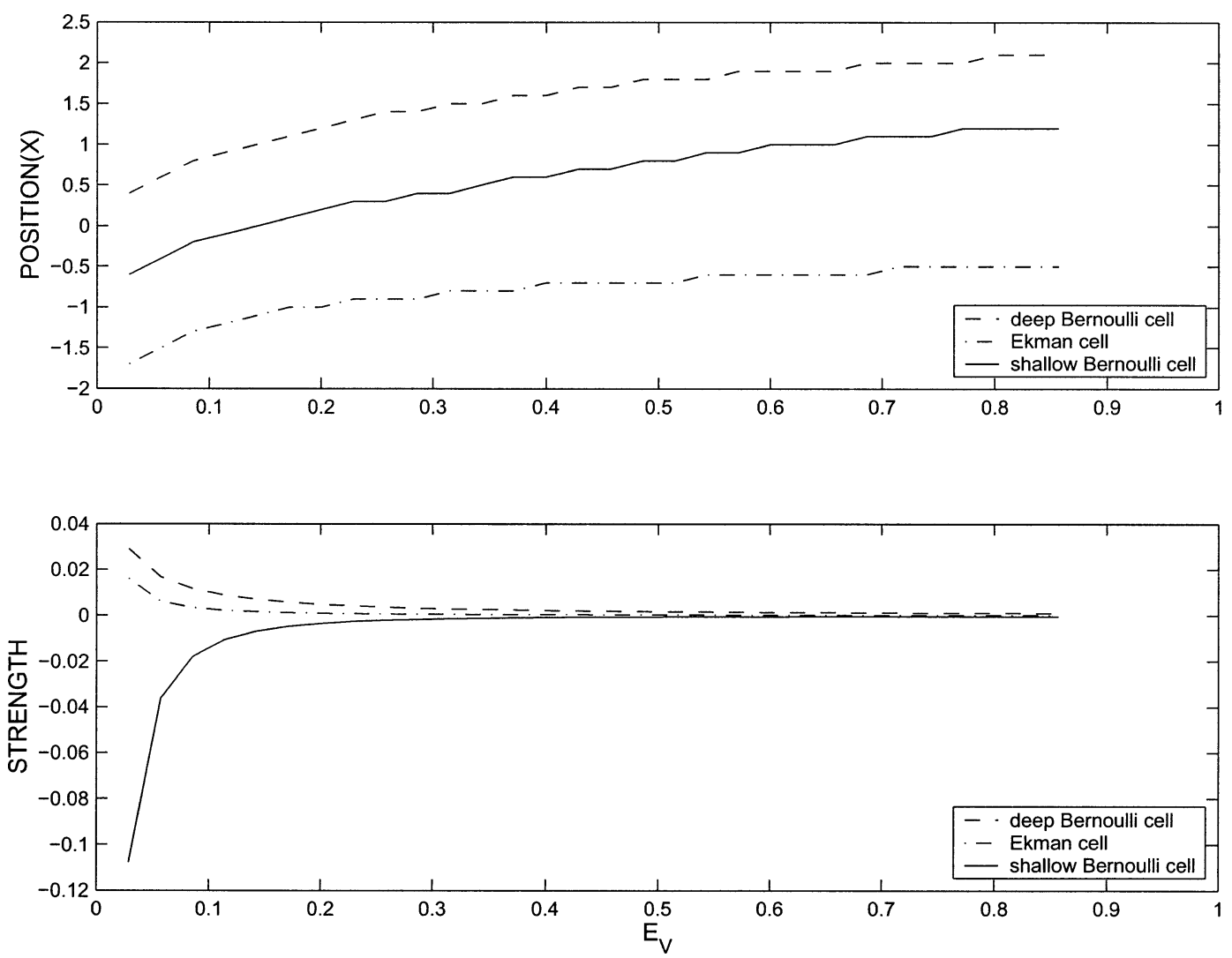

FIG. 4. Locations and strengths of Ekman cell and Bernoulli cells with varying $E$ : (top) location and (bottom) strength. Dashed line is deep Bernoulli cell, dash-dotted line is shallow Bernoulli cell, and solid line is the Ekman cell.

tance of this term is recognized by Loder (1980), and it turns out to constitute an important driving mechanism in our discussion as well. The circulation generated by the term is referred as a "Bernoulli cell."

The third term represents tidal mixing of potential vorticity, which, being dominated by depth change, is negative. Such mixing would induce a negative alongshore flow (Ou 1999), which then drives a circulation with the offshore flow at the bottom and onshore flow at the surface. Since its driving mechanism is similar to the Ekman dynamics associated with the alongshore flow, it is referred as an "Ekman cell."

The final term is the Stokes drift, the dynamics of which have been explained well in the literature (e.g., Longuet-Higgins 1969), and hence it will not be discussed further here.

It is seen that even in the relatively simple case of vertical front subjected to barotropic tides, circulation cells of different signs are generated. Their superimposition thus may lead to complicated circulation patterns depending on their relative strengths. Such hybrid behavior is clearly reflected in the following calculations. By breaking down the circulation into these composition cells of more definite characteristics, however, one hopes to provide a dynamical framework to understand the total pattern.

\section{Analytical solution}

Given the definition

$$
\xi=\frac{z}{\sqrt{2 E_{V}}}
$$

(2.25) has a general solution of the form

$$
\begin{aligned}
\psi= & \exp (\xi)\left(C_{1} \cos \xi+C_{2} \sin \xi\right) \\
& +\exp (-\xi)\left(C_{3} \cos \xi+C_{4} \sin \xi\right)+G(\xi),
\end{aligned}
$$

where $C_{i}(i=1-4)$ are integration constants and $G(\xi)$ is a particular solution:

$$
G(\xi)=\int_{0}^{\xi} y_{1}(\xi-s) \Pi(s) d s,
$$

with

$$
y_{1}(\xi)=\sin (\xi) \cosh (\xi)-\cos (\xi) \sinh (\xi)
$$

and $\Pi$ denoting the right side of (2.25) (see appendix $\mathrm{B}$ for details). The constants are determined by the boundary conditions (see appendix $\mathrm{C}$ for details). With the cross-frontal circulation determined, it is straightforward to calculate the alongfrontal flow and the crossfrontal mean sea surface elevation (Dong 2002).

To examine the parameter dependence of the cross- 

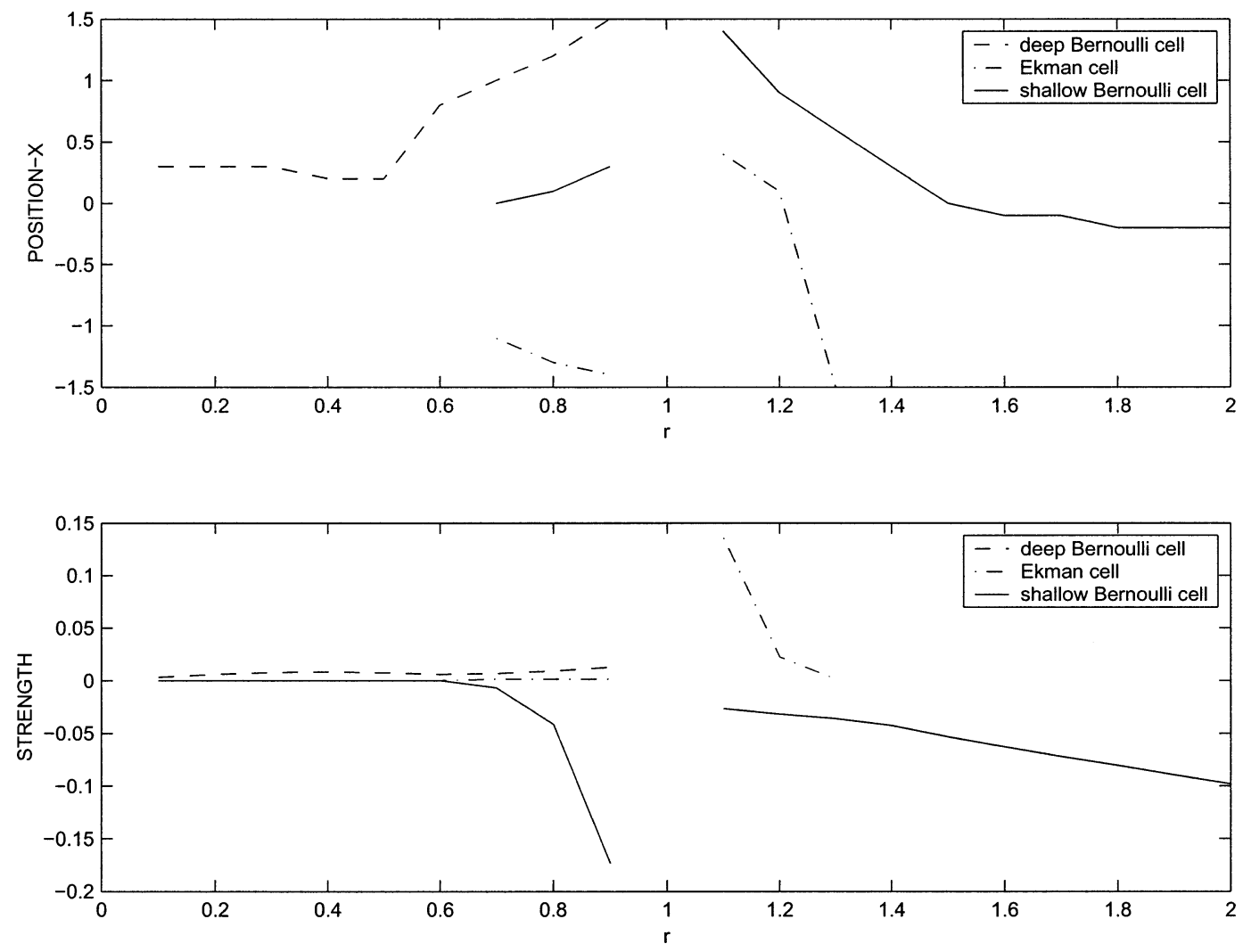

FIG. 5. Same as in Fig. 4, but for varying $r$.

frontal mean circulation, we consider the bottom topography of the exponential form

$$
h=e^{x}, \quad-2 \leq x \leq 2 .
$$

The dependence of the circulation on the form of the bottom topography will be discussed in section 5 .

Because the Stokes drift does not significantly alter the cell structure, its effect on the vertical structure of the circulation is trivial (see section 5), and it is well explored, we assume $M=0$ in the following discussion of the basic cases.

\section{a. Homogenous ocean}

With $B_{U}=0$ and $M=0$, the right side of the equation (2.25) contains only Bernoulli and Ekman terms. As an example, we set $r=0.7, E_{V}=0.15, F=0.3$, which can be representative of the Georges Bank. For example, based on an estimate of the vertical diffusivity (Zimmerman 1986), one may obtain a current scale of 70 $\mathrm{cm} \mathrm{s}^{-1}$ with a depth scale $H_{0}$ of $50 \mathrm{~m}$.

Figure 1a shows the cross-shore streamfunction, which is composed of three cells: a clockwise cell in the middle shelf, sandwiched between two counterclockwise cells. The cell structure is due to the combination of the Ekman and Bernoulli terms as seen below.
The streamfunction driven by the Ekman term is plotted in Fig. 1b, which consists of one counterclockwise cell, and that due to the Bernoulli term consists of one clockwise cell as shown in Fig. 1c. It is noted, moreover, that the centers of two cells are located at about the same location, but the Ekman cell is stronger and narrower than the Bernoulli cell. The linear superposition of the two cells results in the multiple-cell structure as shown in Fig. 1a: the middle cell being referred as Ekman cell and the shallow and deep cells as the shallow and deep Bernoulli cells, respectively. The alongshore mean current is plotted in Fig. 1d, which flows in the direction with the shallow water to the right as expected.

To estimate the dimensional magnitudes of the currents in the above example, one recalls the velocity scale is $70 \mathrm{~cm} \mathrm{~s}^{-1}$, so that one obtains a cross-shore current range from -1.4 to $2.0 \mathrm{~cm} \mathrm{~s}^{-1}$, and the alongshore current is about $30 \mathrm{~cm} \mathrm{~s}^{-1}$.

The basic cell structure is consistent with that of Loder and Wright (1985) except for the clockwise cell in the shallow region, which lies outside their model domain. The results are also comparable with Tee's result (1985) except in the shallow region, where the difference is due to the assumption of the rigid lid $(M=0)$ made in the basic case. The free-surface case $(M \neq 0)$ can be found in section 5 . 

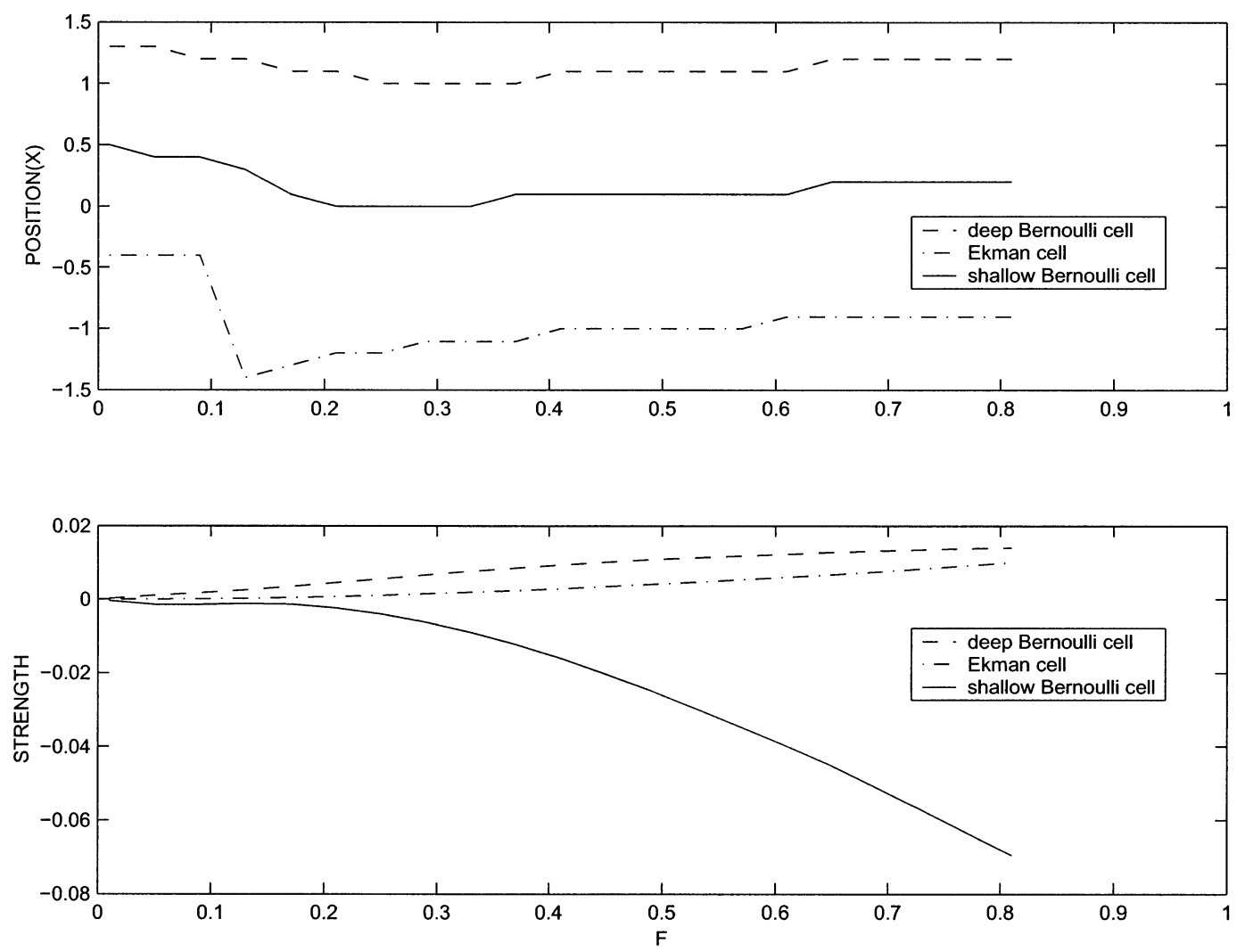

FIG. 6. Same as in Fig. 4, but for varying $F$.

\section{b. Winter front}

In winter, because of the surface cooling and strong wind stress, a tidal front extends from the bottom to the surface. When the density increases offshore, the gravitational force would generate a clockwise cell in the same sense as the Bernoulli cell, whose strength and structure vary with the frontal structure. Ou et al. (2003) have obtained an analytical solution for the winter front, which is used here to calculate the density field, as plotted in Fig. 2a.

The streamfunction associated with the frontal term on the right side of (2.25) is plotted in Fig. 2b. With the inclusion of the Bernoulli and Ekman cells, the streamfunction is shown in Fig. 2c. It is seen that the deep Bernoulli cell is strengthened while the Ekman cell is weakened, which is expected from the superimposition of Fig. 2b and Fig. 1a.

\section{c. Summer front}

In summer, the front is characterized by an inshore vertical mixing and an offshore seasonal stratification, and it intersects both the surface and the bottom. For a representation of the summer, based on Simpson and Hunter (1974) we consider the density field plotted in Fig. 3a.

Again, the streamfunction driven by the density gra- dient is plotted Fig. 3b, which in contrast to the winter front, consists of two cells associated with the two branches: one is the counterclockwise cell in the upper part, and the other is the clockwise cell in the lower part. With the inclusion of the other two terms on the right side of (2.25), the cross-frontal circulation is plotted in Fig. 3c. It is seen that, as expected from Fig. 1a, both the deep Bernoulli cell and the Ekman cell have been strengthened.

\section{Parameter dependence}

Equation (2.25) contains explicitly only two dimensionless parameters $E_{V}$ and $B_{U}$; other parameters-such as $F, M$, and $r$, however-affect the circulation through the tidal stress terms. In this section, the sensitivities of the cross-frontal circulation to these parameters are discussed.

\section{a. Varying $E_{V}$}

We vary $E_{V}$ between 0.03 and 0.9 , which corresponds to vertical viscosity ranging from 0.01 to $0.2 \mathrm{~m}^{2} \mathrm{~s}^{-1}$ at the water depth of $50 \mathrm{~m}$. The $x$ positions of the centers of the cells and their strengths defined as the values of the streamfunction at their centers are plotted in Figs. $4 \mathrm{a}$ and $4 \mathrm{~b}$, respectively. It is seen that all cells move 

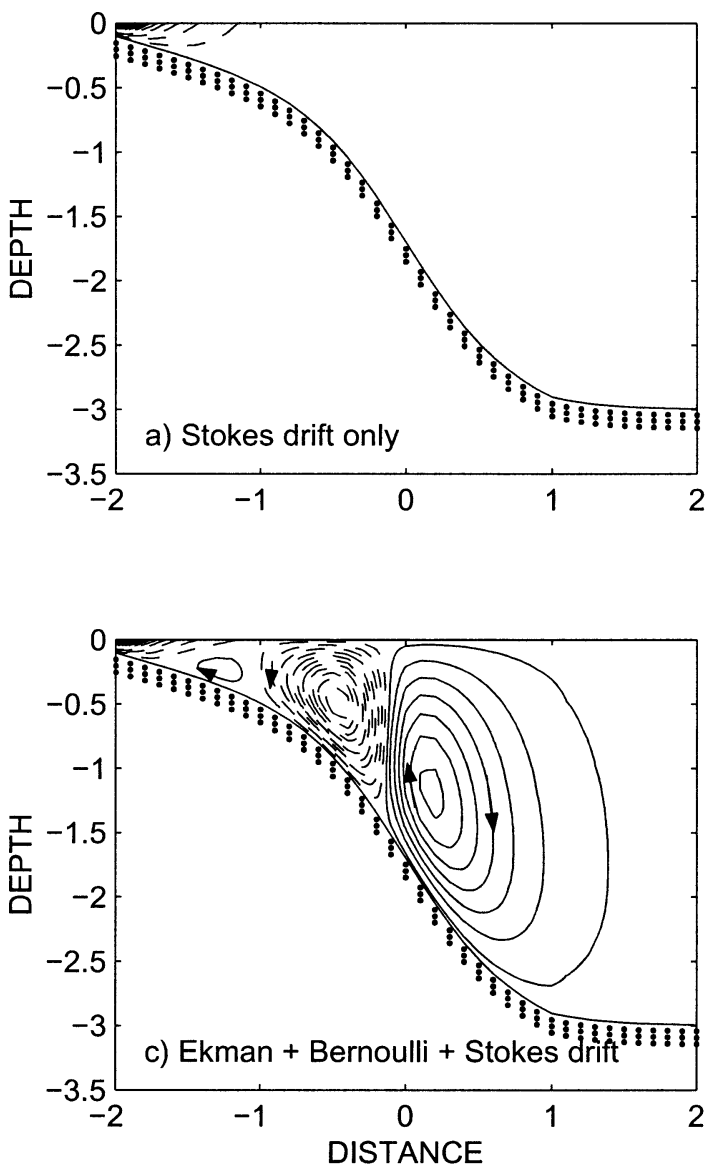

offshore with increasing $E_{V}$. The strengths of all three cells, however, decrease with an increase in $E_{V}$. Because greater $E_{V}$ implies stronger vertical mixing, the above results suggest that increasing vertical mixing would push the cross-shore cells offshore and weaken them in the meantime.

\section{b. Varying $r$}

The dimensionless parameter $r$ is the ratio of the Coriolis parameter to the tidal frequency. For a semidiurnal tide, $r$ ranges from 0 to 1.04 from the equator to the pole. For a diurnal tide, the range is from 0 to 2.08 . Figure 5 shows the dependence of positions and strengths of the cells on the variation in $r$. The singularity case of $r=1$ is not discussed here. When $r$ is smaller than 0.6 , only one Bernoulli cell dominates. When $r$ is between 0.6 and 1.0, three cells exist. Within the range, the Ekman cell strengthens dramatically with increasing $r$ while the two Bernoulli cells do not change their strengths, and both the Ekman cell and the deep Bernoulli cell move offshore while shallow Bernoulli cell moves onshore. For $r$ lies between 1.0 and 1.3, there are only two cells: the shallow Bernoulli cell and the Ekman cell. The deep Bernoulli cell has vanished in this range of $r$. With increasing $r$, both cells move on-

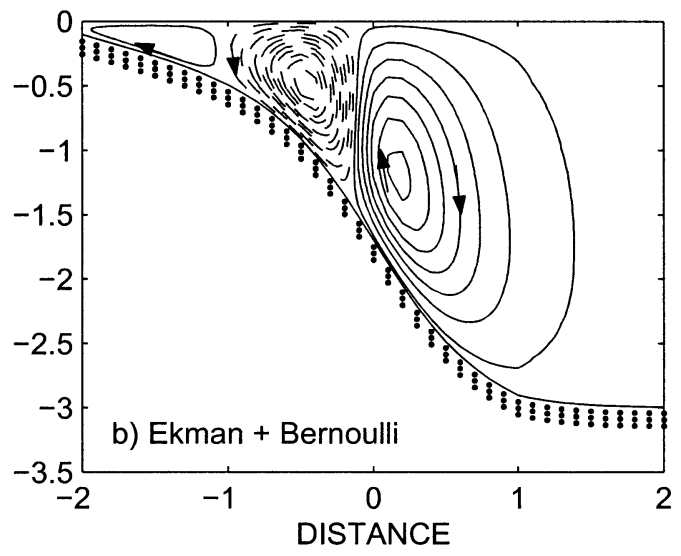

FIG. 7. Streamfunctions with Stokes drift included $(M \neq 0)$ : (a) with only Stokes term; (b) with Ekman and Bernoulli terms only; and (c) with Stokes drift, Ekman, and Bernoulli terms. Contour interval is $2 \times 10^{-3}$, and the dotted lines are for values less than zero.

shore, with the Bernoulli cell weakening and the Ekman cell strengthening. For $r$ greater than 1.3, only the Ekman cell remains.

\section{c. Varying $F$}

This parameter indicates the importance of the bottom friction, which enters both the vertical-averaged tidal equations and boundary conditions. As shown in Fig. 6 , the strengths of all three cells increase with $F$, but the Ekman cell depends on $F$ more than the Bernoulli cells. The locations of cells do not change greatly with changing $F$.

\section{d. Varying $M$}

The parameter $M$, defined in (A.9), measures the importance of the free-surface elevation. Because the tide propagates onshore, the Stokes flow is directed onshore, which necessitates a net offshore Eulerian transport. To compare with Tee (1985), we set $M=6 \times 10^{-4}$, and the topography is slightly different from the basic case. Figure 7a shows the streamfunction associated with only the Stokes drift, the fourth term on the right side of (2.25), which shows an offshore flow. Figure $7 \mathrm{~b}$ shows the streamfunction with the Ekman and Bernoulli terms 

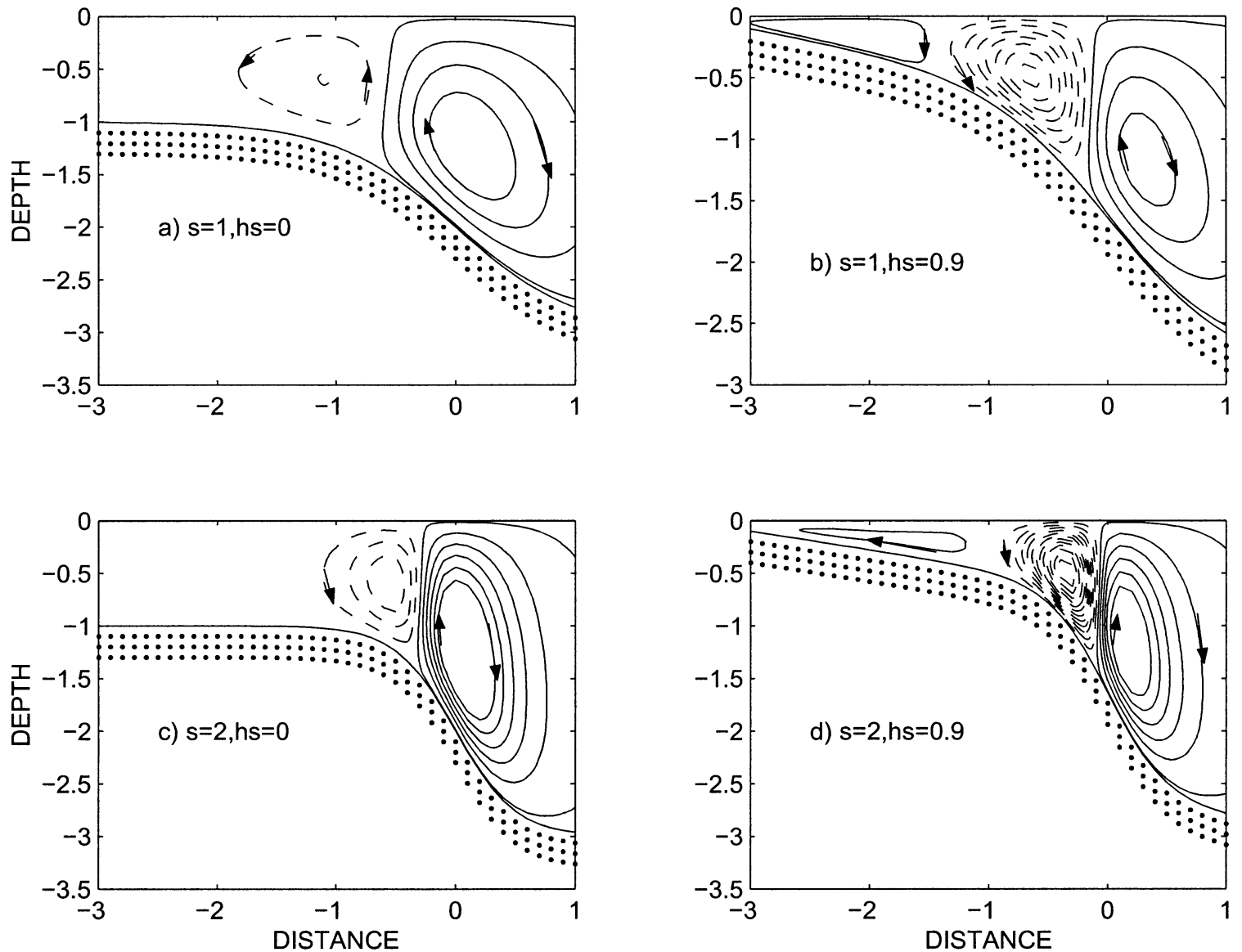

FIG. 8. The streamfunction with different topography in (5.1)-(5.2): (a) $s=1, h_{s}=0$; (b) $s=1, h_{s}=0.9$; (c) $s=2, h_{s}=0$; and (d) $s$ $=2, h_{s}=0.9$. The displayed streamfunction is multiplied by $1 \times 10^{3}$. The contour interval is $0.7 \times 10^{-3}$, and the dotted lines are for values less than zero.

turned on. Figure 7c is the combination of Stokes drift, Ekman, and Bernoulli terms. The result is consistent with Tee's result (his Fig. 5). The smaller $M$ is, the less important the free-surface elevation is and therefore the smaller the magnitude of the Stokes drift is.

\section{e. Varying $B_{U}$}

Because the baroclinic tides are neglected, the Burger Number $B_{U}$ exists only in the equation governing the mean motion. The effect of this parameter on the circulation is straightforward, and hence the result is not plotted. As can be seen from (2.25), increasing $B_{U}$ would enhance the deep Bernoulli cell and weaken the Ekman cell.

\section{f. Varying topography}

In the preceding sections, we use an exponential form of the bottom topography. Does the change of this form affect the cross-shore circulation? Here we consider a different form of bottom topography:

$$
\begin{aligned}
h(x)= & 2+\tanh (s x), \quad \text { for } 0<x<1, \quad \text { and } \\
h(x)= & -h_{S}\left(1-\frac{x}{5}\right) \\
& +[2+\tanh (s x)], \quad \text { for }-5<x<0,
\end{aligned}
$$

where $s$ and $h_{S}$ are adjustable constants.

Figure 8 shows the streamfunctions for four different sets of $\left(s, h_{s}\right)$. Figures $8 \mathrm{a}$ and $8 \mathrm{~b}$ both have $s=1$, with $h_{S}=0$ and 0.9 , respectively. With $h_{S}=0$, the bottom is apparently flat onshore and a shallow Bernoulli cell does not exist because of the absence of the variation of the tidal kinetic energy. With $h_{S}=0.9$, however, the water continues to shoal onshore, and the three-cell structure emerges. The similar response can be found with $s=2$ and $h_{s}=0$ and 0.9 (in Figs. 8c,d), which represents a steeper slope. The steeper slope strengthens and narrows the cells.

\section{Discussion}

Decomposition of the cross-frontal circulation into four components allows one to better understand its 
complex structure. However, the model has constant vertical viscosity and neglects the nonlinear interaction of main flow coupling of the density field to the circulation. The relative magnitude of the four terms may vary with the relaxation of these limitations, which in turn may affect the circulation pattern. A comparison of analytical solution with numerical solutions from a primitive equation model is presented in Dong (2002) and Dong et al. (2003, manuscript submitted to J. Phys. Oceanogr.), in which effects of the nonuniform vertical viscosity and coupling of the density field to the circulation are discussed in detail.

The assumption that all variables are uniform in the alongshore direction precludes an alongshore pressure gradient. The latter implies a tidal wave impinging to the sloping bottom at a right angle, obviously a special case. The effect of the alongshore pressure gradient on the alongshore residual current is discussed in Huthnance (1973) and Loder (1980). Its effect on the crossfrontal mean circulation is an interesting topic that warrants future study.

\section{Summary}

Multiple cells are a common structure of the crossfrontal circulation, as suggested in the previous studies. We derive an equation governing the circulation and then identify and elucidate the forcing mechanisms for generating these cells. The horizontal density gradient associated with a winter front would generate a clockwise frontal cell (facing in the direction with the shallow water to the left). The vertical mixing acting on the mean alongshore flow would generate a clockwise Ekman cell. The cross-shore variation of the tidal kinetic energy would generate a counterclockwise Bernoulli cell. In addition, the Stokes drift would generate a mean Eulerian offshore flow while the tide propagates toward the shallow water. The combination of these cells gives rise to a varied pattern in the cross-frontal circulation. The breakdown of the cross-frontal circulation into its component cells thus provides a dynamical framework for interpreting and understanding its total behavior.

The centers of the Bernoulli cell and Ekman cell are located at almost the same position. However, the Bernoulli cell spans wider than the Ekman cell, and so their superimposition gives rise to the three-cell structure with one Ekman cell at the middle shelf and two Bernoulli cells on its two sides. We consider cases of homogenous ocean, winter and summer fronts, respectively. For a homogenous ocean, the circulation is dominated by the three cells. For the winter front, the offshore Bernoulli cell is strengthened. For the summer front, the two opposite cells are found vertically because of the two branches of the summer front.

The dependence of the analytical solution on key model parameters is investigated. It is found that increasing $E_{V}$ would displace all cells offshore while their strengths are weakened; increasing $F$ would strengthen the Ekman cell. Only within a special range of $r$ can three cells be found; the cross-shore variations in $E_{V}$ and $F$ do not change the circulation pattern significantly.

Acknowledgments. This research is supported by the National Science Foundation under Grant ATM-961820. We thank Robert Houghton, Arnold Golden, and Robin Robertson from Lamont-Doherty Earth Observatory of Columbia University; Robert Wilson from State University of New York at Stony Brook; and George Mellor, Leo Oey, and Tal Ezer from Princeton University for their valuable discussions and comments. Special thanks are given to two anonymous reviewers for their constructive comments.

\section{APPENDIX A}

\section{Tidal Model}

Following Wright and Loder (1985), nondimensionalized equations that govern vertical-averaged tidal fields are

$$
\begin{aligned}
\frac{\partial U^{\prime}}{\partial t}-r V^{\prime} & =-\frac{\partial \eta^{\prime}}{\partial x}-\frac{r F U^{\prime}}{h}, \\
\frac{\partial V^{\prime}}{\partial t}+r U^{\prime} & =-\frac{r F V^{\prime}}{h}, \text { and } \\
M \frac{\partial \eta^{\prime}}{\partial t}+\frac{\partial\left(h U^{\prime}\right)}{\partial x} & =0,
\end{aligned}
$$

and the nondimensionized equations that govern tidal fields with vertical structure are

$$
\begin{aligned}
\frac{\partial u^{\prime}}{\partial t}-r v^{\prime} & =-\frac{\partial \eta^{\prime}}{\partial x}+r E_{V} \frac{\partial^{2} u^{\prime}}{\partial z^{2}}, \\
\frac{\partial v^{\prime}}{\partial t}+r u^{\prime} & =r E_{V} \frac{\partial^{2} v^{\prime}}{\partial z^{2}}, \quad \text { and } \\
\frac{\partial u^{\prime}}{\partial x}+\frac{\partial w^{\prime}}{\partial z} & =0
\end{aligned}
$$

where uppercase denotes vertical averaging and lowercase denotes vertical-varied variables. In the above equations, the dimensionless parameters are defined as the following:

$$
\begin{aligned}
r & =\frac{f}{\sigma}, \\
F & =\frac{k}{f H_{0}}, \\
M & =\left(\frac{L}{L_{W}}\right)^{2}, \\
L_{W} & =\frac{\sqrt{H_{0} g}}{\sigma}, \text { and }
\end{aligned}
$$




$$
E_{V}=\frac{\nu}{f H_{0}^{2}}
$$

The tidal model applied here is linear with no tidal and mean current interaction. Its solution has been discussed in Wright and Loder (1985) with the assumption of a rigid lid, namely, $M=0$. When $M$ is big enough and the first term on the right side of (A.3) cannot be neglected, a numerical solution is obtained for the vertical-averaged tidal equations (Dong 2002). The constant vertical eddy viscosity is used.

The purpose of introducing the set of vertically averaged equations is to obtain the surface elevation gradient that constitutes the forcing for (A.4)-(A.6).

The lateral boundary conditions for equations (A.1)(A.3) are

$$
U^{\prime}=U_{b}^{\prime} \quad \text { and } \quad \eta^{\prime}=\eta_{b}^{\prime},
$$

where $U_{b}^{\prime}$ is time-dependent cross-shore current and $\eta_{b}^{\prime}$ is sea surface elevation at the boundaries, and both of them are specified to be consistent with an onshore progressive wave. The boundary conditions for (A.4)(A.6) are, at $z=0$,

$$
\begin{aligned}
\frac{\partial\left(u^{\prime}, v^{\prime}\right)}{\partial z} & =0 \text { and } \\
w^{\prime} & =\frac{\partial \eta^{\prime}}{\partial t}, \quad \text { and },
\end{aligned}
$$

at $z=-h$,

$$
\begin{aligned}
\frac{\partial\left(u^{\prime}, v^{\prime}\right)}{\partial z} & =\frac{F}{E_{V}}\left(u^{\prime}, v^{\prime}\right) \text { and } \\
w^{\prime} & =-u^{\prime} \frac{\partial \eta^{\prime}}{\partial x} .
\end{aligned}
$$

\section{APPENDIX B}

\section{Particular Solution to (2.25)}

For a fourth-order ordinary differential equation

$$
\frac{d^{4} \psi}{d z^{4}}+a_{3} \frac{d^{3} \psi}{d z^{3}}+a_{2} \frac{d^{2} \psi}{d z_{2}}+a_{1} \frac{d \psi}{d z}=f(z),
$$

the particular solution is

$$
Y(z)=\int_{0}^{z} y(z-\xi) f(\xi) d \xi
$$

where $y$ is the solution to the homogenous equation (B.1) with the boundary condition

$y=\frac{d y}{d z}=\frac{d^{2} y}{d z^{2}}=0$ and $\quad \frac{d^{3} y}{d z_{3}}=1 \quad$ at $z=0$.

The particular solution $Y$ satisfies

$$
Y=\frac{d Y}{d z}=\frac{d^{2} Y}{d z^{2}}=\frac{d^{3} Y}{d z^{3}}=0 \quad \text { at } z=0 .
$$

\section{APPENDIX C}

\section{Integration Constants of (4.2)}

Applying the boundary conditions (2.31) and (2.32) to determine constants in (4.2), we obtain the linear algebra equations as follows:

$$
\mathbf{A C}=\mathbf{D},
$$

where $\mathbf{A}$ is the coefficient matrix

with

$$
\mathbf{A}=\left[\begin{array}{cccc}
0 & 1 & 0 & -1 \\
1 & 0 & 1 & 0 \\
-a_{0} a_{1}+\left(a_{0}-2\right) a_{2} & \left(2-a_{0}\right) a_{1}-a_{0} a_{2} & a_{0} a_{3}+\left(2+a_{0}\right) a_{4} & -\left(2+a_{0}\right) a_{3}+a_{0} a_{4} \\
a_{1} & a_{2} & a_{3} & a_{4}
\end{array}\right]
$$

$$
\begin{gathered}
\xi_{0}=\frac{h}{\sqrt{2 E_{V}}}, \\
\mathbf{C}=\left[\begin{array}{l}
C_{1} \\
C_{2} \\
C_{3} \\
C_{4}
\end{array}\right], \quad \text { and }
\end{gathered}
$$

$a_{1}=e^{\xi_{0}} \cos \left(\xi_{0}\right)$,

$a_{2}=e^{\xi_{0}} \sin \left(\xi_{0}\right)$,

$a_{3}=e^{-\xi_{0}} \cos \left(\xi_{0}\right)$, 


$$
\mathbf{D}=\left[\begin{array}{c}
-\left.\frac{1}{2} \frac{d^{2} G}{d \xi^{2}}\right|_{\xi=0} \\
Q_{s}-\left.G(\xi)\right|_{\xi=0} \\
-\frac{d^{2} G}{d \xi^{2}}+\left.a_{0} \frac{d G}{d \xi}\right|_{\xi=\xi_{0}} \\
-G\left(\xi_{0}\right)
\end{array}\right] .
$$

Because it is easily shown that

$$
\operatorname{Det}(\mathbf{A}) \neq 0,
$$

there is a nontrivial solution given by

$$
\mathbf{C}=\mathbf{A}^{-1} \mathbf{D} \text {. }
$$

\section{REFERENCES}

Chen, C., and R. C. Beardsley, 1995a: A numerical study of stratified tidal rectification over finite-amplitude banks. Part I: Symmetric bank. J. Phys. Oceanogr., 25, 2090-2110.

$\longrightarrow$, and $-1995 \mathrm{~b}$ : A numerical study of stratified tidal rectification over finite-amplitude banks. Part II: Georges Bank. J. Phys. Oceanogr., 25, 2111-2128.

— change over a finite amplitude asymmetric bank: A model study with application to Georges Bank. J. Mar. Res., 56, 1163-1201.

Dong, C., 2002: Tidally induced cross-frontal mean circulation. Ph.D. thesis, Columbia University, $109 \mathrm{pp}$.

Greenberg, D. A., 1983: Modeling the mean barotropic circulation in the Bay of Fundy and Gulf of Maine. J. Phys. Oceanogr., 13, 886-904.
Hill, A. E., and Coauthors, 1993: Dynamics of tidal mixing fronts in the North Sea. Philos. Trans. Roy. Soc. London, 343, 431-446.

Houghton, R., and C. Ho, 2001: Diapycnal flow through the Georges Bank tidal front: A dye tracer study. Geophys. Res. Lett., 28, $33-36$.

Huthnance, J. M., 1973: Tidal current asymmetries over the Norfolk sandbanks. Estuarine Coastal Mar. Sci., 1, 89-99.

Loder, J. W., 1980: Topographic rectification of tidal currents on the sides of Georges Bank. J. Phys. Oceanogr., 10, 1399-1416.

—_ and D. G. Wright, 1985: Tidal rectification and frontal circulation on the sides of Georges Bank. J. Mar. Res., 43, 581-604.

— , and D. Brickman, 1992: Detailed structure of currents and hydrography on the northern side of Georges Bank. J. Geophys. Res., 97, 14 331-14 351.

- K. F. Drinkwater, N. S. Oakey, and E. P. Horne, 1993: Circulation, hydrographic structure and mixing at tidal fronts: The view from Georges Bank. Philos. Trans. Roy. Soc. London, 343, 447-460.

Longuet-Higgins, M. S., 1969: On the transport of mass by timevarying ocean currents. Deep-Sea Res., 16, 431-447.

Ou, H.-W., 1999: A model of tidal rectification by potential vorticity mixing. Part I: Homogeneous ocean. J. Phys. Oceanogr., 29, 821-827.

_- 2000: A model of tidal rectification by potential vorticity mixing. Part II: Frontal regime. J. Phys. Oceanogr., 30, 564-571.

— C. C. Dong, and C. Chen, 2003: Tidal diffusivity: A mechanism for frontogenesis. J. Phys. Oceanogr., 33, 840-847.

Simpson, J. H., and J. R. Hunter, 1974: Fronts in the Irish Sea. Nature, 250, 404-406.

Tee, K. T., 1985: Depth-dependent studies of tidally induced residual currents on the sides of Georges Bank. J. Phys. Oceanogr., 15, $1818-1846$.

Wright, D., and J. W. Loder, 1985: A depth-dependent study of the topographic rectification of tidal currents. Geophys. Astrophys. Fluid Dyn., 31, 169-220.

Zimmerman, J. T. F., 1986: The tidal whirlpool: A review of horizontal dispersion by tidal and residual currents. Neth. J. Sea. Res., 20, 133-154. 